\title{
EXTENDED MALCEV DOMAINS
}

\section{R. E. JOHNSON}

In 1936, Malcev [1] constructed a cancellative monoid $M$ generated by eight elements which couldn't be embedded in a group. Later on, Chehata [2] and Vinogradov [3] showed that $M$ could be ordered. This gave a counterexample to the conjecture that every ordered monoid could be embedded in a group. Since the monoid ring $F[M]$ of an ordered monoid $M$ over a (not-necessarily commutative) field $F$ is a (not-necessarily commutative) integral domain, this also showed that not every integral domain could be embedded in a field. In the present paper, we give a somewhat different construction of an ordered monoid $N$ generated by six or more elements which cannot be embedded in a group.

Let $Z$ denote the ring of integers, $S=\left\{x_{i j} \mid i=1,2, j \in Z\right\}$ be a set of indeterminates, and $A$ be the free monoid generated by $S$. Each $a \in S, a \neq 1$, has the form $a=a_{1} \cdots a_{n}$ for some $a_{i} \in S$. We call $n$ the degree of $a, n=\operatorname{deg} a$. As usual, we let $\operatorname{deg} 1=0$. We order $A$ by degrees and lexicographically from the assumed ordering

$$
1<x_{1 i}<x_{1 j}<x_{2 i}<x_{2 j} \quad \text { for all } i, j \in Z \text { with } i<j \text {. }
$$

Thus, if $a=a_{1} \cdots a_{m}$ and $b=b_{1} \cdots b_{n}$ with $a_{i}, b_{i} \in S$, then $a<b$ iff either $\operatorname{deg} a<\operatorname{deg} b$ or $\operatorname{deg} a=\operatorname{deg} b$ and there exists an integer $k$ such that $a_{i}=b_{i}$ if $i<k$ and $a_{k}<b_{k}$.

For any $i, j \in Z$, let $[i, j]$ and $[i, j]^{\prime}$ be defined as follows: (1) $[i, j]$ $=[i, j]^{\prime}=(i+j) / 2$ if $i+j$ is even, (2) $[i, j]=(i+j-1) / 2$ and $[i, j]^{\prime}$ $=(i+j+1) / 2$ if $i+j$ is odd. Also, let $T \subset A$ be defined by

$$
T=\left\{x_{2 i} x_{1 j} \mid i, j \in Z, i>j\right\}
$$

and $B$ be the ideal of $A$ generated by $T$. Finally, let $N=A-B$.

We define an operation of multiplication, $\cdot$, in $N$ by letting $a \cdot b=a b$ if $a b \notin B$, and

$$
x_{2 i} \cdot x_{1 j}=x_{2 k} x_{1 k^{\prime}} \quad \text { if } i>j, \text { where } k=[i, j] .
$$

Thus, if $a=a^{\prime} x_{2 i}$ and $b=x_{1 j} b^{\prime}$, with $i>j$ and $a^{\prime}, b^{\prime} \in N$, we have $a \cdot b$ $=a^{\prime} x_{2 k} x_{1 k^{\prime}} b^{\prime}$. Since nothing more happens in $a \cdot b$ than the replacement of one $x_{2 i}$ on the right end of $a$ by $x_{2 k}$ and one $x_{1 j}$ on the left end of $b$ by $x_{1 k^{\prime}}$, evidently multiplication in $N$ is associative and $\operatorname{deg} a \cdot b$ $=\operatorname{deg} a+\operatorname{deg} b$ for all $a, b \in N$.

Received by the editors April 19, 1968. 
Each $a \in N, a \neq 1$, has a unique normal form $a=a_{1} \cdots a_{n}$ where all $a_{i} \in S$ and $a_{i} a_{i+1} \notin T, i=1, \cdots, n-1$. We order $N$ by assuming its elements, in normal form, are ordered by the ordering in $A$.

THEOREM 1. $\{N ; \cdot\}$ is an ordered monoid.

Proof. Let $a, b, c \in N$ with $a<b$. If $\operatorname{deg} a<\operatorname{deg} b$, then clearly $a \cdot c<b \cdot c$ and $c \cdot a<c \cdot b$. So let us assume that $\operatorname{deg} a=\operatorname{deg} b=n$ and $c \neq 1$.

Case 1. $n>1$. By assumption, $a=a_{1} \cdots a_{n}, b=b_{1} \cdots b_{n}, c=c_{1}$ $\cdots c_{k}$, where $a_{i}, b_{i}, c_{i} \in S$, and there exists an integer $j$ such that $a_{i}=b_{i}$ if $i<j$ and $a_{j}<b_{j}$. If $j=1$, evidently $a \cdot c<b \cdot c$, and we need only check that $c_{k} \cdot a_{1}<c_{k} \cdot b_{1}$ to prove that $c \cdot a<c \cdot b$. If $1<j<n, c_{k} \cdot a_{1}=c_{k} \cdot b_{1}$ and hence $c \cdot a<c \cdot b$; and obviously $a \cdot c<b \cdot c$. If $j=n, c_{k} \cdot a_{1}=c_{k} \cdot b_{1}$ and $c \cdot a<c \cdot b$; while $a \cdot c<b \cdot c$ provided $a_{n} \cdot c_{1}<b_{n} \cdot c_{1}$. This reduces the problem to the following case.

Case 2. $n=1$, with $a, b, c \in S$. If $a=x_{1 i}$ and $b=x_{1 j}$, with $i<j$, then $a \cdot c=a c<b c=b \cdot c$. Also, $c \cdot a=c a<c b=c \cdot b$ unless $c=x_{2 m}$ and $m>i$. If $i<m \leqq j$, then $c \cdot a=x_{2 k} x_{1 k^{\prime}}<x_{2 m} x_{1 j}=c \cdot b$ since $k=[m, i]<m$. If $m>j$, $c \cdot a=x_{2 k} x_{1 k^{\prime}}<x_{2 l} x_{1 l^{\prime}}$ because either $k=[m, i]<[m, j]=l$ or $k=l$ and $k^{\prime}<l^{\prime}$ (this is the case only if $j=i+1$ and $m+i$ is even).

If $a=x_{2 i}$ and $b=x_{2 j}$, with $i<j$, then a similar analysis shows that $c \cdot a<c \cdot b$ and $a \cdot c<b \cdot c$ for all $c \in S$.

Finally, we might have $a=x_{1 i}$ and $b=x_{2 j}$. Then $a \cdot c<b \cdot c$ for all $c \in S$. If $c=x_{1 m}$ then clearly $c \cdot a=c a<c b=c \cdot b$. If $c=x_{2 m}$, then $c \cdot a$ $=c a<c b=c \cdot b$ if $m \leqq i$, whereas, if $m>i, c \cdot a=x_{2 k} x_{1 k^{\prime}}<x_{2 m} x_{2 j}=c \cdot b$ because $k=[m, i]<m$. This proves the theorem.

For any $m, n \in Z$, with $m<n$, let $S(m, n)=\left\{x_{i j} \mid i=1,2, m \leqq j \leqq n\right\}$ and $N(m, n)$ be the submonoid of $N$ generated by $S(m, n)$. Since $i \leqq[i, j] \leqq[i, j]^{\prime} \leqq j$ for all $i, j \in Z$ with $i<j$, evidently each element of $N(m, n)$ when expressed in normal form is a product of elements of $S(m, n)$. If $m<n, m^{\prime}<n^{\prime}$, and $n-m=n^{\prime}-m^{\prime}$, then it is clear that $N(m, n) \cong N\left(m^{\prime}, n^{\prime}\right)$.

THEOREM 2. The monoid $N(1,3)$ generated by six elements cannot be embedded in a group.

The standard proof of Malcev can be used. Thus, assume that $N(1,3)$ is a submonoid of some group $G$. Since $x_{22} \cdot x_{11}=x_{21} x_{12}, x_{23} \cdot x_{11}$ $=x_{22} x_{12}$, and $x_{23} \cdot x_{12}=x_{22} x_{13}$, we have in $G$ that $x_{22}^{-1} x_{21}=x_{11} x_{12}^{-1}=x_{23}^{-1} x_{22}$ $=x_{12} x_{13}^{-1}$. Hence, $x_{22} x_{12}=x_{21} x_{13}$ contrary to the definition of the monoid $N$. Therefore, $N(1,3)$ cannot be embedded in a group.

A consequence of Theorem 2 and our remarks above is that the monoid $N(m, n)$ with $n>m+1$ cannot be embedded in a group. 
If $F$ is any integral domain, then the monoid ring $F[N]$ generated by $N$ over $F$ is also an integral domain. For if $f=f_{1} a_{1}+\cdots+f_{m} a_{m}$ and $g=g_{1} b_{1}+\cdots+g_{n} b_{n}$ are elements of $F[N]$, with $f_{i}, g_{i} \in F, f_{m} \neq 0$, $g_{n} \neq 0$, and $a_{i}, b_{i} \in N$ such that $a_{1}<\cdots<a_{m}$ and $b_{1}<\cdots<b_{n}$, then $f g$ is nonzero with highest term $f_{m} g_{n} a_{m} b_{n}$. Since $N$ cannot be embedded in a group, $F[N]$ cannot be embedded in a field. This is also true of each subdomain $F[N(m, n)]$ for which $n>m+1$.

For any integral domain $D$ and its associated ring $(D)_{n}$ of $n \times n$ matrices over $D$, the poset $P$ of annihilating right ideals of $(D)_{n}$ has dimension at least $n$. If $D$ is a field, or a subring of a field, the dimension of $P$ is exactly $n$. For other domains, the dimension of $P$ might be considerably different as the following result shows.

Theorem 3. If $F$ is a field, $R=F[N]$, and $P$ is the poset of annihilating right ideals of $(R)_{2}$, then $P$ satisfies neither the dcc nor the acc.

Proof. Let $\left\{e_{i j} \mid i, j=1,2\right\}$ be the usual unit matrices in $(R)_{2}$. If $i+1 \geqq j$ and $r+1 \geqq s$, then $x_{2 i} \cdot x_{1 j}=x_{2 r} \cdot x_{18}$ iff $i+j=r+s$. For each $n \in Z$, let $a_{n}=x_{2 n} e_{11}-x_{2 n+1} e_{12}$ and $b_{n j}=x_{1 n+1} e_{1 j}+x_{1 n} e_{2 j}, j=1$, 2. Then the right annihilator of $a_{n}$ in $(R)_{2}$ is given by

$$
\left(a_{n}\right)^{r}=\sum_{k=-\infty}^{n} \sum_{j=1}^{2} b_{k j}(R)_{2}
$$

For if $\sum f_{i j} e_{i j} \in\left(a_{n}\right)^{r}$, then $x_{2 n} f_{11}=x_{2 n+1} f_{21}$ and $x_{2 n} f_{12}=x_{2 n+1} f_{22}$. If $f_{i j}=p_{i j 1} c_{i j 1}+p_{i j 2} c_{i j 2}+\cdots$, with $p_{i j k} \in F, \quad c_{i j k} \in N$, and $c_{i j 1}<c_{i j 2}$ $<\cdots$, necessarily $p_{11 k}=p_{21 k}, p_{12 k}=p_{22 k}, x_{2 n} \cdot c_{11 k}=x_{2 n+1} \cdot c_{21 k}, x_{2 n} \cdot c_{12 k}$ $=x_{2 n+1} \cdot c_{22 k}$ for each $k$. Thus, it is clear that $c_{11 k}=x_{1 i} \cdot c_{11 k}^{\prime}, c_{21 k}=x_{1 j} \cdot c_{21 k}^{\prime}$ with $i \leqq n+1, j=i-1$, and $c_{11 k}^{\prime}=c_{21 k}^{\prime}$ for each $k$; and similarly for $c_{12 k}$ and $c_{22 k}$. Thus, $\sum f_{i j} e_{i j}$ has the form given in (1). Since $\left(a_{n}\right)^{r}<\left(a_{n+1}\right)^{r}$ for all $n \in Z, P$ satisfies neither the $d c c$ nor the acc. This proves the theorem.

\section{BIBLIOGRAPHY}

1. A. I. Malcev, On the immersion of an algebraic ring into a field, Math. Ann. 113 (1936), 686-691.

2. C. G. Chehata, On an ordered semigroup, J. London Math. Soc. 28 (1953), 353356.

3. A. A. Vinogradov, On the theory of ordered semigroups, Ivanov. Gos. Ped. Inst. Uc. Zap. Fiz.-Mat. Nauki 4 (1953), 19-21. (Russian)

UNIVERSITY OF NEW HAMPSHIRE 